> Qu'il le déplore ou s'en réjouisse, chacun s'accorde aujourd'hui sur un constat: il n'est guère de développement pérenne envisageable des biotechnologies sans partage. Tel médicament n'aura pu être développé que grâce au gène de résistance isolé de telle plante malgache. Tel chercheur ne pourra mettre au point son test de dépistage d'une maladie génétique qu'à partir des échantillons biologiques fournis par des patients donneurs. Et ce n'est qu'en accédant aux résultats du séquençage du génome du riz que la recherche pourra mettre au point des espèces plus performantes. Parce qu'en la matière, il n'y a pas d'innovation possible sans la double contribution de la ressource biologique et de la technique, le développement des biotechnologies débouche inéluctablement sur un impératif de partage entre ceux qui fournissent la ressource, ceux qui la travaillent et ceux qui l'exploitent. <

\section{Partage des biotechnologies : le contrat comme avant-garde}

Christine Noiville

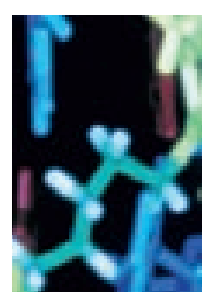

\section{Le contrat comme instrument de partage}

Contre toute attente, c'est à ce jour le contrat (entendu comme l'accord de deux ou plusieurs personnes ayant pour objet et effet de créer des obligations) qui, mieux que la loi (au sens générique du terme), paraît aboutir à mettre en œuvre cet impératif. Une telle affirmation sera probablement de nature à faire frémir ceux pour qui le contrat, particulièrement dans un domaine jeune comme celui des biotechnologies, est synonyme de liberté débridée, de pillage des faibles par les forts, ou encore de satisfaction d'intérêts privés au détriment de l'intérêt général. Elle constitue pourtant l'une des lignes de force les plus marquantes en la matière : non seulement les contrats se révèlent pertinents pour coordonner les prétentions respectives des différents acteurs (donneurs d'échantillons biologiques humains, fournisseurs de ressources microbiennes ou végétales, chercheurs, biobanques, industriels...) et prévenir les conflits d'intérêts qui peuvent les opposer, mais l'outil contractuel prend ici des allures d'avant-garde. Parce qu'il offre à chacun la faculté de laisser parler son imagination et de façonner une relation juridique (dans le respect de l'ordre public), les parties y échafaudent des règles originales destinées à limiter l'exclusivisme et à organiser les modalités du nécessaire partage : celui des ressources biologiques et des connaissances qui leur sont associées, d'une part, et celui de ce qu'il est désormais convenu d'appeler les bienfaits, d'autre part. 


\section{Partage des ressources et des connaissances}

Dans ce domaine, une quantité croissante de figures contractuelles visent à limiter les exclusivités bloquantes et autres formes de maîtrise qui enserrent la matière. La multiplication des brevets dans le domaine des biotechnologies, leur enchevêtrement et l'étendue généralement large des monopoles conférés, parfois sur les outils même de la recherche (séquences de gènes, lignées cellulaires...), ont placé les chercheurs dans une position délicate: ils ont désormais à franchir de multiples «péages», en amont, pour obtenir les outils de recherche, comme en aval, pour utiliser ou exploiter un résultat [4]. Leur situation est encore compliquée par la stratégie adoptée par certains titulaires pour exploiter leurs brevets: l'exemple des brevets détenus par l'entreprise Myriad Genetics sur certains gènes de prédisposition au cancer du sein (BRCA) est emblématique [5], mais d'autres exemples, moins visibles, illustrent un phénomène similaire. C'est le cas des clauses dites de rétroconcession, par lesquelles une entreprise ou un institut de recherche met à la disposition de la communauté scientifique une séquence d'ADN, un modèle animal transgénique ou encore les données issues du séquençage d'un génome, mais s'assure en contrepartie sinon de la titularité des inventions que le chercheur mettra au point grâce à cet outil, au moins d'une licence (souvent exclusive) d'exploitation de ces inventions. Or ce mécanisme de rétroconcession se révèle très préjudiciable à la recherche: en raison d'un emploi récurrent de ce type de clauses dans les contrats les liant aux entreprises, les universités américaines, notamment, n'ont plus aujourd'hui qu'une maîtrise très limitée des innovations biotechnologiques qu'elles ont pourtant massivement contribué à développer $[6,7]$. Une forte poussée d'exclusivisme favorisée par une politique très libérale d'octroi de brevets : cette réalité est telle qu'elle paraît aboutir à une sorte d'effet boomerang que deux auteurs américains ont qualifié de «tragédie des anti-communs » [8].

C'est précisément pour déjouer certains de ces blocages que les ressources du contrat sont de plus en plus utilisées, non pas tant pour rejeter le brevet, que pour adapter la brevetabilité des inventions biotechnologiques aux exigences de partage. Par contrat, les parties conçoivent et adaptent le droit des brevets, de telle sorte que ce dernier s'exerce à l'appui de cet impératif. Trois exemples sont caractéristiques à cet égard.

\section{Les pools de brevets}

En eux-mêmes, les pools de brevets n'ont rien de véritablement nouveau. Ils ont, de longue date, été mis en place par les entreprises pour regrouper tout ou partie de leurs portefeuilles de brevets, s'accorder les uns aux autres des licences croisées sur certains titres stratégiques et neutraliser ainsi les brevets « de blocage». Or dans le domaine des biotechnologies, cette technique connaît une fortune certaine au-delà du monde industriel, dans celui de la recherche publique, et c'est là qu'est son originalité.

La Public initiative for intellectual property resource for agriculture (PIPRA) est exemplaire de cette tendance: plusieurs institutions publiques américaines spécialisées en biotechnologies végétales ont, par contrat, institué un système de gestion collective de leurs brevets. L'intérêt est double: entre les partenaires du contrat, un tel regroupement de brevets permet de faciliter l'accès aux technologies brevetées, chacun des contractants s'engageant en effet à mettre ses technologies à la disposition des autres; vis-à-vis des tiers, et particulièrement de l'industrie, la mise en commun des brevets accroît la force de négociation des institutions publiques et, ce faisant, leur permet non seulement d'accéder plus aisément aux innovations des entreprises, mais aussi de mieux valoriser leurs propres brevets. Le contrat prévoit notamment que les chercheurs restent propriétaires de leurs brevets, la licence d'exploitation, quand elle est accordée, étant soigneusement limitée dans le temps et l'espace : ils ont dès lors la possibilité de réutiliser leurs inventions ultérieurement.

\section{Le contrat Génoplante}

Un deuxième exemple, plus novateur bien que bâti sur un même principe de mutualisation, est fourni par le contrat Génoplante. Cet accord, qui lie aussi bien des instituts de recherche que des entreprises privées, a pour objectif de développer des recherches fondamentales et appliquées en biotechnologies végétales. Afin de donner un maximum d'impulsion à la recherche et éviter les blocages, les parties ont imaginé un montage schématiquement articulé autour de deux pôles : il est d'abord institué un système de mise en «pot commun » de différentes ressources et techniques, de sorte que chaque partenaire a la garantie d'accéder aux ressources et technologies des autres lorsqu'elles sont utiles à la collaboration; dans un second temps, la titularité et l'exploitation des futurs résultats s'organise selon trois règles.

Les partenaires s'engagent d'abord à ce que certaines des données acquises (très précisément les connaissances fondamentales, dont il est important de favoriser la publication et l'échange) soient mises dans le domaine public. Il est ensuite convenu que les organismes publics de recherche sont seuls propriétaires des brevets portant sur des «résultats génériques » qui, bien que non valorisables à court terme, sont à la fois essentiels à la recherche et susceptibles de déboucher sur une large gamme d'applications. Enfin, qu'ils soient ou non protégés par un droit de propriété industrielle, tous les résultats obéissent à la règle de l'accès garanti : en pratique, chacun peut ainsi prendre connaissance, en temps réel, de l'ensemble des données, et bénéficier alors de résultats issus de travaux dans lesquels il n'a pas nécessairement été directement impliqué. Mieux encore, chaque partenaire peut librement et gratuitement utiliser ces résultats pour ses recherches 
propres, même si c'est à des fins de recherche appliquée: l'Inra, l'un des contractants de Génoplante, est ainsi autorisé à utiliser sans frais, dans le cadre de ses programmes de recherche de nouvelles variétés, des brevets protégeant des inventions développées par d'autres. Le travail de sélection ne se retrouve pas bloqué quand les variétés employées sont brevetées et, une fois sa nouvelle variété développée, l'Inra aura l'assurance de pouvoir la faire protéger par un droit de propriété intellectuelle, et pourra la commercialiser.

Par le biais du contrat, c'est donc de façon assez subtile que s'articulent ici deux exigences, la réservation d'un côté et, de l'autre, l'accès aux échantillons, aux données et aux résultats. Le ressort de ce principe de mutualisation n'est ni béatitude naïve, ni pur altruisme: le brevet n'est pas rejeté (il reste le cœur du système) et la contribution de chacun aux différentes étapes du partenariat (apports initiaux, contribution aux résultats de la recherche...) n'est jamais niée. II s'agit simplement de combiner finement des impératifs souvent considérés comme antinomiques: mise en commun/maintien d'un certain degré de concurrence et exclusivité/diffusion des résultats. On ne saurait certes passer sous silence les tensions pouvant, en pratique, naître de la mise en œuvre d'un tel montage : il en est ainsi du principe de mise dans le domaine public de certains résultats qui, à l'épreuve du temps, paraît s'effilocher, certains partenaires étant enclins, par précaution stratégique, à se réserver l'ensemble des données produites. Cependant, avec des contrats de ce type, on pénètre dans un univers contractuel très sophistiqué mettant au point des droits à intensité variable, destinés à s'approprier sans toutefois les bloquer les ressources biologiques et les données qui leur sont attachées. C'est en cela que ce type de contrat peut corriger les effets pervers de l'exclusivisme.

\section{Contrats open source ou open access}

Un troisième exemple de contrat, plus audacieux encore, pousse plus loin cette logique: il s'agit des contrats inspirés de la philosophie open source ou open access, développée il y a vingt ans en matière de logiciels informatiques. On pensera notamment à cette licence d'accès établie par le consortium de séquençage HapMap, dans le domaine de la génétique humaine, ou à la Bios public licence, établie par le Center for the application of molecular biology to international agriculture (Cambia) en Australie. Dans les deux cas, une même idée structure le contrat: HapMap et Cambia mettent à la disposition des chercheurs ou des entreprises des données ou des outils de recherche; cette mise à disposition est non exclusive ; le licencié s'engage à ne solliciter aucun droit de propriété industrielle sur aucune des entités transférées; il peut déposer des demandes de brevets sur une application, à la condition toutefois de ne pas englober, dans les revendications, les entités elles-mêmes; enfin, il s'engage à ne transférer ces dernières qu'à des tiers assujettis à ces mêmes obligations.
À la manière des licences libres type linux, ces contrats cherchent ainsi à articuler l'intérêt des inventeurs, dont le droit de solliciter un brevet sur une application est acquis, tout comme celui d'être protégé de la contrefaçon, celui des chercheurs, libres de puiser dans un vaste pool de ressources restant en libre accès, et ceux de la société, qui devrait gagner à un tel montage a priori favorable à l'innovation.

\section{Partage des bienfaits}

Conçu comme un moyen de façonner les conditions d'octroi, le champ et les modalités d'exploitation du brevet, et mettre en œuvre l'impératif de partage des connaissances, le contrat assume une fonction similaire s'agissant du partage des bienfaits.

Le problème est bien connu: il s'agit de faire en sorte que le développement des biotechnologies ne bénéficie pas seulement à ceux qui ont développé les inventions, mais aussi à ceux qui en ont fourni la matière première. Cette exigence se présente sous un jour très différent selon qu'il s'agit de matériel humain ou non: alors qu'elle doit, dans le second cas, prendre la forme d'un partage d'avantages avec les pays fournisseurs (Convention sur la diversité biologique), il en va tout autrement dans le premier cas, puisque le droit français postule un principe de gratuité (Loi de bioéthique) empêchant, à quelques rares exceptions près, toute rémunération du don d'échantillons biologiques. Néanmoins, dans un cas comme dans l'autre, c'est bien le contrat qui se dessine comme l'instrument privilégié du partage.

S'agissant de la biodiversité, ce recours au contrat a été voulu par les rédacteurs de la Convention sur la diversité biologique. Il a pour rôle précis d'organiser les conditions d'accès aux ressources biologiques: paiement d'un droit de collecte (exclusif ou non), rémunération éventuelle des échantillons, titularité et conditions d'exploitation des futurs brevets par le prospecteur, montant des redevances au profit du fournisseur... Loin d'être évacuées du contrat de bioprospection, les clauses relatives à la propriété industrielle en constituent ainsi la pierre angulaire, car ce sont elles qui, entre autres, déterminent les modalités et l'étendue du retour d'avantages. Toute la question est d'apprécier, contrat par contrat, l'efficacité des montages ainsi opérés. Ainsi, comment juger concrètement le caractère équitable de l'échange lorsque le champ est envahi par une rhétorique parfois trompeuse, qui oblige à savoir garder ses distances: par combien d' «effets d'annonce» les parties disent vouloir poursuivre, par leurs contrats, des objectifs si vastes qu'ils incluent la satisfaction de l'humanité tout entière? L'évolution des modèles contractuels observables et la jeunesse de la matière empêchent en outre toute généralisation hâtive, et contraignent à un raisonnement partiellement spéculatif et largement provisoire. Enfin, la variété des situations est grande, et si certains contrats paraissent se 
situer à la hauteur des ambitions (clauses de futures redevances, clauses permettant au fournisseur de contrôler les travaux de prospection et de recherche de son co-contractant...), d'autres laissent plus perplexe. II n'empêche, par sa souplesse, sa force d'adaptation et la liberté qu'il offre aux parties d'inventer, le contrat apporte une contribution décisive à une politique d'échange équitable de biodiversité.

Qu'un phénomène similaire se produise en matière de biotechnologies humaines paraissait peu probable en France où le droit, en postulant la gratuité en la matière, n'offre au donneur aucune autre alternative que de consentir au don ou de s'y opposer. Pourtant, tout l'intérêt d'un certain nombre de pratiques contractuelles récentes consiste précisément à dépasser cette alternative pour instituer entre donneurs, chercheurs et médecins une relation destinée sinon à faire profiter les premiers des avantages escomptés, au moins à éviter que l'exploitation des ressources ne nuise à leurs intérêts. Un exemple, à cet égard emblématique, est celui d'un contrat conclu entre l'association américaine PXE International, regroupant des patients atteints par une maladie génétique rare (le pseudoxanthoma elasticum) et l'Université de Hawaï : le contrat s'attache à prévoir les conditions de prélèvement et de circulation du matériel prélevé, mais aussi les conditions auxquelles les futures inventions seront exploitées. C'est sur ce dernier point, précisément, que le contrat innove: il prévoit ainsi que l'association de patients devient cotitulaire des brevets délivrés. Certes, cela exige que l'association soit co-inventeur (ce qui suppose qu'elle ait activement participé au développement de l'invention) et qu'elle s'acquitte des taxes nécessaires à l'entretien des brevets, alors même que leur exploitation commerciale reste aléatoire. Mais par le biais de ce contrat, l'association se ménage aussi une puissante faculté de contrôle: cotitulaire du brevet, elle peut obtenir une rémunération sur son éventuelle exploitation, mais aussi acquérir et surtout maîtriser l'exercice des droits de propriété intellectuelle (contrôle des développements commerciaux, marge de manœuvre à l'égard de la politique commerciale de ses co-contractants...).

Rien n'empêche d'imaginer que ce contrat de droit américain puisse valoir en droit français, et devenir l'outil d'un véritable partenariat entre patients et chercheurs.

\section{Conclusions}

Le contrat comme outil de partage des ressources, des connaissances, des bienfaits: qu'on ne s'y trompe pas, il ne s'agit pas de prétendre que la matière puisse être abandonnée à la liberté contractuelle et le temps du contrat n'a pas purement et simplement remplacé celui de la loi [9]. Le soutien de la loi se révèle évidemment indispensable à une bonne politique juridique : c'est ainsi son rôle de redistribuer la donne de l'innovation en luttant contre les exclusivités bloquantes, comme le législateur français s'est du reste attaché à le faire en limitant le champ des brevets sur les gènes ou en étendant celui des licences d'office. De la même manière, si subtil que soit le contrat échafaudé par l'association PXE International, il ne saurait constituer une alternative généralisable, la plupart des associations de patients n'ayant pas les moyens d'imposer un tel équilibre contractuel. Imaginons un instant des malades du cancer prétendant subordonner leur participation à une recherche biomédicale à l'inscription, dans le protocole, d'une clause d'accès privilégié aux futurs médicaments : il y a fort à parier que le promoteur les exclurait de la cohorte... La réalité oblige en effet à constater que les sujets d'expérimentation, généralement isolés, non organisés collectivement et malades, ne négocient pas un protocole d'expérimentation, ils y consentent ou pas, ils y entrent ou non, rien de plus. Toutefois, l'enjeu de la loi ne saurait voiler un constat : celui du relais qu'est susceptible d'assumer le contrat pour mettre en œuvre cet impératif, désormais décisif, qu'est le partage dans le domaine des biotechnologies. $\diamond$

\section{SUMMARY}

Sharing of biotechnology: contractual agreements as avant-garde

In the face of growing exclusivist strategies that tend to hamper research and undermine equity, sharing imperatives have become a cornerstone of biotechnology development. Whereas a proliferation of laws and conventions now require the sharing of biological resources and knowledge as well as the sharing of benefits, contractual agreements more and more appear as a most useful tool to implement these requirements. $\diamond$

\section{RÉFÉRENCES}

1. Bellivier $F$, Noiville $C$. La circulation du vivant humain : modèle de la propriété ou modèle du contrat? In : Revet $\mathrm{T}$ (sous la direction de). Code civil et modèles. Des modèles du Code au Code comme modèle. Bibliothèque de l'Institut André Tunc. Paris: Presses de la Sorbonne, 2005 : 101.

2. CCNE. Problèmes éthiques posés par les collections de matériel biologique et les données d'information associées: «biobanques», «biothèques ». Avis $n^{\circ} 77$, 20 mars 2003.

3. UNESCO. Déclaration internationale sur les données génétiques humaines, 16 octobre 2003.

4. Clayes A. Rapport sur les conséquences des modes d'appropriation du vivant sur les plans économique, juridique et éthique. Paris: Office parlementaire d'évaluation des choix scientifiques et technologiques, 2004.

5. Cassier M, Stoppat-Lyonnet D. L'opposition contre les brevets de Myriad Genetics et leur révocation totale ou partielle en Europe : premiers enseignements. Med Sci (Paris) $2005 ; 6-7: 658-62$.

6. Weil A (sous la direction de). Vers une mutualisation européenne de la propriété intellectuelle publique en biotechnologies à vocation agronomique. Paris, 2004.

7. Rai AK, Eisenberg RS. Bayh-Dole reform and the progress of biomedecine. Law and contemporary problems. Winter-Spring, $2003: 289$.

8. Heller MA, Eisenberg RS. Can patents deter innovation? The anticommons in biomedical research. Science 1998 ; $280: 698-701$.

9. Bellivier F, Noiville C. Contrats et vivant. Essai sur la circulation juridique des ressources biologiques. Paris: LGDJ, 2005 (sous presse). 